Ю. М. Селиванов, д-р техн. наук., С. С. Кузенный

\title{
СОВРЕМЕННЫЕ ИНТЕРФЕРЕНЦИОННО-ОПТИЧЕСКИЕ ТЕХНОЛОГИИ ИССЛЕДОВАНИЯ И НЕРАЗРУШАЮЩЕГО КОНТРОЛЯ НЕОДНОРОДНЫХ КОНСТРУКЦИЙ (ОБЗОР)
}

Рассмотрено состояние технологий исследования и неразрушающего контроля прочностных и динамических свойств неоднородных конструкций на основе голографической интерферометрии, спекл-интерферометрии, высококогерентной и низкокогерентной классической интерферометрии. Приведены их особенности, области эффективного применения.

Ключевые слова: голографрическая интерферометрия, спеклинтерферометрия, классическая интерферометрия, новые технологии, области применения.

Введение. Обеспечение прочности, несущей способности и других свойств конструкций и их элементов, особенно при наличии в них различных неоднородностей, остается в числе актуальных и чрезвычайно сложных проблем. В последние десятилетия в исследованиях и неразрушающем контроле неоднородных конструкций возрастающее применение находят технологии, преимущественно цифровые, основанные на интерференционно-оптических методах - голографической интерферометрии (ГИ), спекл-интерферометрии (СИ), высококогерентной и низкокогерентной классической интерферометрии [1 - 24]. Использование указанных методов позволяет бесконтактно получать наглядную качественную и достаточно точную количественную информацию о статических и нестационарных полях микро- и наноперемещений поверхности контролируемого объекта под влиянием различных факторов. Далее по этим перемещениям можно давать оценку напряженнодеформированного состояния (НДС) объекта, выявлять его конструктивные несовершенства, технологические дефекты, эксплуатационные повреждения, прослеживать развитие трещин и др.

В обзоре представлены некоторые новые разработки в этой области, а также состояние соответствующих динамично развивающихся технологий.

Голографические технологии. Голографическая интерферометрия считается наиболее развитым и широко используемым способом исследования и неразрушающего контроля неоднородных тонкостенных конструкций. Под этим способом понимаются методы сравнения двух или более когерентных волновых фронтов, несущих информацию об исследуемом объекте, из которых, по крайней мере, один волновой фронт получен с использованием голографии, то есть посредством ре- 
гистрации и последующего восстановления амплитудно-фазового распределения волнового фрронта [1, 14, 19]. Уникальными достоинства голографических методов являются возможность одновременного наблюдения поверхности площадью 10 кв. м. и более, а также отсутствие принципиальных ограничений на форму, состояние поверхности и материал испытываемого объекта.

Основы анализа голографических интерферограмм. Если различие между сравниваемыми состояниями объекта вполне описывается некоторой векторной функцией перемещений его поверхности $\bar{L}=\bar{L}(x, y, z, t)$, то для освещенности $I=I(x, y, z, t)$ наблюдаемого на голографической интерферограмме изображения объекта в общем случае справедливо выражение [1]

$$
I \approx I_{0}\left|\frac{1}{T} \int_{t_{0}}^{t_{0}+T} \exp \left[-i \int_{t_{0}}^{t}(\bar{K} \cdot \bar{V}(\tau)) d \tau\right] d t\right|^{2},
$$

где $I_{0}=I_{0}(x, y, z)$ - освещенность изображения объекта в стационарном состоянии; $T$ - длительность экспозиции; $t$ и $\tau \leq t$ - время; $t_{0}$ - время начала экспонирования; $i$ - мнимая единица; $\bar{V}(\tau)=\bar{V}(x, y, z, t)$ - скорость движения поверхности во время экспонирования; $\bar{K}=\bar{K}(x, y, z)-$ вектор чувствительности оптической схемы, который по направлению совпадает с биссектрисой угла $2 \theta$ между направлениями освещения и наблюдения точки поверхности, а величина его равна $|\bar{K}|=(4 \pi \cdot \cos \theta) / \lambda ; \lambda$ - длина волны используемого излучения.

Внутренний интеграл в (1) определяет перемещение поверхности на текущий момент $t$. Подмодульное выражение в целом описывает интерференцию между оптическими полями, которые соответствуют всем положениям поверхности в процессе экспонирования, и зависит от метода получения интерферограммы. Очевидно, что центры темных интерференционных полос объединяют точки поверхности объекта, где выполняется условие $I=\min$, а центры светлых полос - точки, где выполняется условие $I=\max$. После вычисления интеграла (1) при одном из этих условий получается уравнение, которое является основой для определения перемещений поверхности объекта по интерферограмме. Оно включает усредненное по времени приращение вектора перемещений $\bar{L}$ и имеет корни, прямо связанные с порядковыми номерами интерференционных полос $N$.

При использовании одной пары значений $\bar{K}$ и $N$ это уравнение позволяет получить одну составляющую вектора перемещений в точке его проекцию на направление вектора $\bar{K}$. Если этого недостаточно для 
оценки НДС объекта, необходимо для каждой точки получить две и более пар значений $\bar{K}$ и $N$ и затем по ним определить величину вектора перемещений, его проекции на координатные оси, деформации и напряжения.

В исследованиях и неразрушающем контроле чаще всего применяются двухэкспозиционная ГИ (метод двойной экспозиции, двухимпульсный метод) и ГИ с усреднением во времени (метод усреднения во времени, метод Пауэлла - Стетсона). Реже используются стробоскопическая ГИ (стробоголографический метод) и ГИ в реальном масштабе времени (реально-временной метод). Возрастающее применение находят метод комбинированных голографических интерферограмм и цифровая ГИ.

Теоретическое обоснование, техника эксперимента, аппаратурное оснащение, интерпретация опытных данных, особенности практического применения методов ГИ обстоятельно изложены в соответствующих многочисленных публикациях [1 - 3, 5, 6, 9, 14, 15, 19 - 22]. Методы ГИ продолжают развиваться и совершенствоваться, находят новые практические приложения. Остановимся на некоторых новых разработках в области голографических технологий.

Анализ интерферограмм по приведенной плотности полос. Известно, что количественная интерпретация интерферограмм позволяет получить наиболее полную информацию о НДС конструкции, что достигается путем определения по интерферограммам трех компонент $u, v$ и $w$ вектора перемещения $\bar{L}$. Однако, в настоящее время отсутствуют универсальные средства автоматизации этого процесса, что является сдерживающим фрактором для широкого использования количественной интерпретации. Кроме того, необходимо решать проблему определения оптимального параметра сглаживания при решении некорректной задачи - аппроксимации дискретных данных, получаемых с погрешностью в результате расшифровки интерферограмм.

Качественная интерпретация интерферограмм по простоте и оперативности существенно превосходит количественный подход и может быть весьма эффективной при выявлении и оценке непосредственно неоднородностей НДС контролируемого объекта. При этом анализируется плотность (густота) интерференционных полос, сопровождающих аномалии поверхностных деформаций нагружаемого объекта в зонах неоднородности НДС. Высокая точность результатов исследований таким способом достигается лишь в отдельных случаях, когда влиянием формы поверхности исследуемого образца, параметров оптической схемы его освещения и наблюдения на плотность полос можно пренебречь. Как правило, это - центральная зона интерферограммы. В периферийных зонах интерференционной картины результаты чаще всего неудовлетворительные.

В исследованиях НДС конструкций наибольший интерес представляют не полные поля деформаций и напряжений, а лишь зоны их экстремумов. По этим зонам судят о концентраторах напряжений конструкции, ее несовершенствах и дефектах, наиболее вероятной картине начала 
разрушения, прочности и других свойствах, а также определяют направления шагов оптимизационных доработок. Рассматриваемый ниже подход к анализу голографических интерферограмм позволяет существенно повысить эффективность выявления и оценки экстремумов механического НДС неоднородной оболочечной конструкции.

Суть данного подхода заключается в том, что при оценке $i$-ой неоднородности НДС контролируемой конструкции сравниваются не максимальные значения плотности полос $\left(\rho_{i}\right)$, то есть градиенты поля прогибов на отдельных участках исследуемой поверхности, как при обычной качественной интерпретации интерферограмм, но непосредственно максимальные линейные деформации этих участков [3].

Для этого на тестовой голографической интерферограмме контролируемого образца, как обычно, находят зоны экстремумов плотности интерференционных полос, то есть места локализации неоднородностей НДС конструкции; в этих зонах регистрируют величину и направление максимальной местной плотности полос $-\rho_{i \max } и \alpha_{i}$.

Затем находится приведенная величина максимальной плотности полос в $i$ - ой зоне $\rho_{i \max }^{*}$. С этой целью значения $\rho_{i \max }$ делят на местный оптико-геометрический коэффицциент $k_{i}$, который учитывает форму исследуемой поверхности, параметры ее освещения и наблюдения и в общем случае определяется по формуле $k_{i}=1 /(\cos \theta \cdot \cos \psi)$, где $\psi-$ угол между векторами $\bar{L}$ и $\bar{K}$ в заданной точке.

Для цилиндрической оболочки под внутренним давлением при ее исследовании с использованием оптической схемы центрального освещения и наблюдения коэффициент $k_{i}$ по длине оболочки остается практически постоянным ( $\left.k_{i} \approx 1\right)$, но в ее окружном направлении, а также по поверхности сорерической оболочки изменяется от $k_{i}=1$ (в центре) до $k_{i}=3,5$ и более (в периферийной зоне).

Далее вдоль направления $\rho_{i \max }^{*}$ вычисляется относительная величина изменения шага $h$ полос $\left(\overline{\Delta h_{i}}=\left|h_{j}-h_{j+1}\right| / h_{j+1}\right.$, где $j$ - номер интерфе-ренционной полосы), то есть абсолютное значение максимальной линейной деформации.

Оценка неоднородности НДС дается по величинам $\rho_{i \max }^{*}, \alpha_{i}, \overline{\Delta h_{i}}$.

Представленный подход сочетает преимущества качественного и количественного анализа интерферограмм, так как предполагает сравнительно быстрое определение всех потенциально важных (с экстремумами НДС) зон конструкции и несложный анализ плотности полос интерференционной картины только в этих квазиплоских зонах с приведенными (одинаковыми) оптико-геометрическими параметрами. Таким образом, 
при приемлемой простоте реализации подход обеспечивает надежные как в центре, так и на периферии интерферограммы результаты, которые по точности приближаются к данным более трудоемкой количественной интерпретации. Очевидно, несложно увеличить эффективность подхода путем автоматизации обработки полос в потенциально важных зонах.

На основе анализа интерферограмм по приведенной плотности полос созданы три взаимодополняющие голографические методики диагностики показателей разрушения оболочечных элементов в предположении применимости деформационного критерия прочности [3, 4]. Преимущество этих методик по сравнению с существующими состоит в сочетании простоты прогнозирования и повышенной достоверности получаемых данных о наиболее деформируемой (напряженной) зоне, направлении максимальных деформаций и ориентации начальной макротрещины сложных оболочечных элементов конструкций из стеклопластика, углепластика и других, подобных им по деформационно-прочностным свойствам материалов.

Метод комбинированных голографических интерферограмм. Состояние голографических исследований и контроля в силу специфики используемых методов не позволяет удовлетворить растущие потребности практики проектирования, изготовления и дальнейшей эксплуатации сложных промышленных объектов в условиях нестационарного нагружения. В частности, использование двухэкспозиционной ГИ требует применения мощных и соответственно дорогостоящих лазеров импульсного или непрерывного излучения. ГИ с усреднением во времени эффективна только при исследовании стационарных (гармонических) колебаний и т.п.

Суть рассматриваемого метода состоит в экспонировании двух состояний контролируемой поверхности: неподвижного (статического) в течение времени $T_{C}=\alpha T$, где коэффрициент $\alpha \leq 1$, и подвижного - в течение времени $T_{\text {д }}=(1-\alpha) T$; тогда, очевидно, $T_{C}+T_{\text {д }}=T-$ продолжительность экспозиции. При таком экспонировании выражение для освещенности точки поверхности на ее восстановленном изображении (1) принимает вид [15]

$$
I \approx I_{0}\left[\alpha+\frac{1}{T} \int_{t_{0}}^{t_{0}+T(1-\alpha)} \exp \left[-i \int_{t_{0}}^{t}(\bar{K} \cdot \bar{V}(\tau)) d \tau\right] d t\right]^{2} .
$$

Используя соотношение (2), путем аналитического или численного интегрирования можно получить формулы и графики для освещенности изображения поверхности на комбинированной интерферограмме, которые посредством параметра $X$ (функции амплитуды или скорости, других параметров движения) связывают картину полос на интерферограмме с полем перемещений поверхности при различных законах ее движения во время голографирования [3, 15]. 
Так, при движении поверхности по закону $\bar{L}(x, y, z, t)=\bar{A} e^{-\beta t}$ или $\bar{L}(x, y, z, t)=\bar{A}\left(1-e^{-\beta t}\right)$, где $\bar{A}=\bar{A}(x, y, z, t)$ - амплитуда, из (2) получается

$$
I \approx I_{0}\left|\alpha^{2}-\frac{2 \alpha}{\beta T}\left\{\Omega_{1} \cos \left(X \cdot \kappa_{1}\right)+\Omega_{2} \sin \left(X \cdot \kappa_{1}\right)\right\}+\frac{1}{(\beta T)^{2}}\left[\Omega_{1}^{2}+\Omega_{2}^{2}\right]\right|,
$$

где $\Omega_{1}=\operatorname{Ci}\left(X \cdot \kappa_{2}\right)-C i\left(X \cdot \kappa_{1}\right) ; \quad \Omega_{2}=\operatorname{Si}\left(X \cdot \kappa_{2}\right)-\operatorname{Si}\left(X \cdot \kappa_{1}\right) ; \quad \operatorname{Ci}(\ldots) \quad$ і $S i(\cdots)$ - интегральный косинус и синус; $\kappa_{1}=e^{-\beta t_{0}} ; \kappa_{2}=e^{-\beta\left[t_{0}+T(1-\alpha)\right]} ;$ $X=\bar{K} \cdot \bar{A}$.

Графрики функции (3) при различных значениях коэффрициента $\alpha$ показаны на рис. 1.

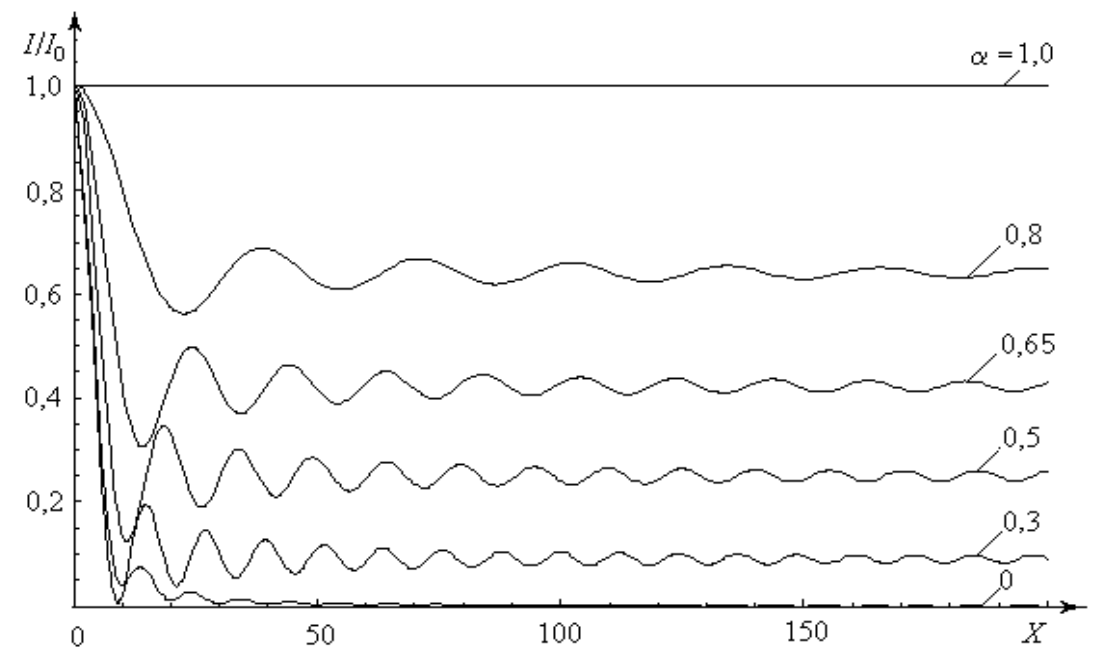

Рис. 1 - Расчетные графики освещенности точки поверхности,

движущейся по экспоненциальной зависимости ( $t_{0}=5$ c, $T=45$ с, $\beta=0,029$ ), на комбинированной интерферограмме

Из графиков, построенных с использованием соотношения (2), следует, что максимальная яркость интерференционных полос убывает с ростом параметра $X$. Общее количество порядков различающихся полос существенно зависит от величины коэффрициента $\alpha$. Причем на графике, соответствующем $\alpha=0,5 \pm 0,1$, различается максимальное количество полос. Кроме того, при увеличении $\alpha$ чувствительность метода к перемещениям (величина, обратная шагу полос) заметно снижается. 
Характерный результат использования метода комбинированных голографических интерферограмм в диагностических исследованиях нестационарных состояний неоднородных конструкций представлен на рис. 2.

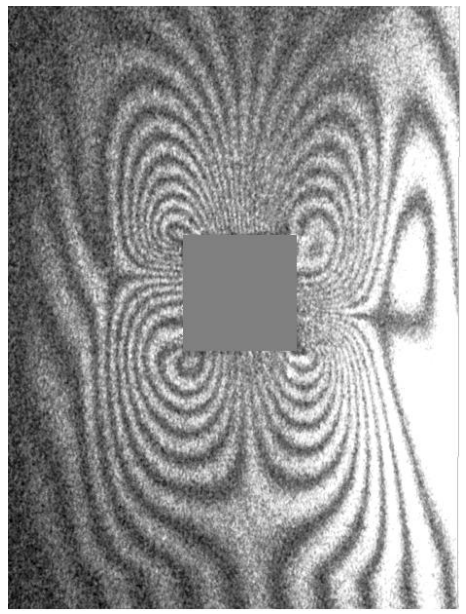

Рис. 2 - Комбинированная интерферограмма стальной оболочки, нагретой до $60^{\circ} \mathrm{C}$, при свободном охлаждении до $25^{\circ} \mathrm{C}$.

Алгоритм расшифровки комбинированных интерферограмм состоит в следующем. Пусть закон движения наблюдаемой поверхности определен, например $\bar{L}(x, y, z, t)=\bar{A} e^{-\beta t}$, вектор чувствительности $\bar{K}$ в каждой ее точке известен, с помощью фрормулы (3) построен расчетный график освещенности точки на комбинированной интерферограмме, соответствующий этому закону и параметрам эксперимента. Величину вектора в произвольной точке $M$ поверхности можно установить с использованием (2) на любой момент времени $t_{*}$ следующим образом.

По интерферограмме определяется порядок интерференционной полосы в заданной точке $\left(\xi_{M}\right)$, и по графику находится соответствующее значение аргумента $\left(X_{M}\right)$.

Поскольку $X=\bar{K} \cdot \bar{A}=|\bar{K}| \cdot|\bar{A}| \cos \psi$ и величина $|\bar{K}|$ известна, то по значению $X_{M}$, с учетом закона движения поверхности, вычисляется величина амплитуды перемещения точки

$$
\left|\bar{A}_{M}\right|=\frac{\lambda}{4 \pi \cos \theta \cos \psi} X_{M},
$$

а затем - и величина вектора перемещения в заданный момент времени $t_{*}$

$$
\left|\bar{L}_{M}\left(t_{1}\right)\right|=\left|\bar{A}_{M}\right| e^{-\beta t_{*}} .
$$


В формуле (4) $\psi$ - угол между векторами $\bar{A}$ и $\bar{K}$.

Результаты апробации [3] показывают, что метод может успешно применяться для бесконтактного измерения нестационарных полей поверхностных деформаций сложных неоднородных элементов конструкций при воздействии на них тепловых полей, механических нагрузок, других фракторов и, таким образом, существенно расширяет прикладные возможности ГИ. Его применение дает возможность получать высокоинформативные качественные и количественные данные о поле неустановившихся перемещений контролируемого объекта на любой момент его десоормирования, используя для этого лазеры непрерывного излучения небольшой выходной мощности (вместо традиционно применяемых дорогостоящих мощных источников) и, соответственно, относительно недорогие компактные голографические измерительные установки.

Методы цифровой голографической интерферометрии. Для методов классической (аналоговой) ГИ при высоком качестве интерферограмм характерны сравнительно узкие диапазоны измеряемых микроперемещений и чувствительности, значительные трудозатраты при регистрации и особенно при обработке интерферограмм, наличие высокоразрешающих регистрирующих сред и др. Это соответственно ограничивает практическое использование классической ГИ в измерениях. С появлением высокоразрешающих цифровых видеокамер технического зрения в мире начался постепенный переход к цифровым методам ГИ, которые в настоящее время развиваются и все шире используются в дефектоскопии и диагностике технических малогабаритных объектов, вытесняя аналоговые методы ГИ [2, 6, 16, 20, 21].

Суть цифровой ГИ заключается в том, что регистрация голограмм осуществляется на матричный светочувствительный сенсор (рис. 3), и полученные таким образом изображения (цифровые голограммы) фриксируются в памяти компьютера.

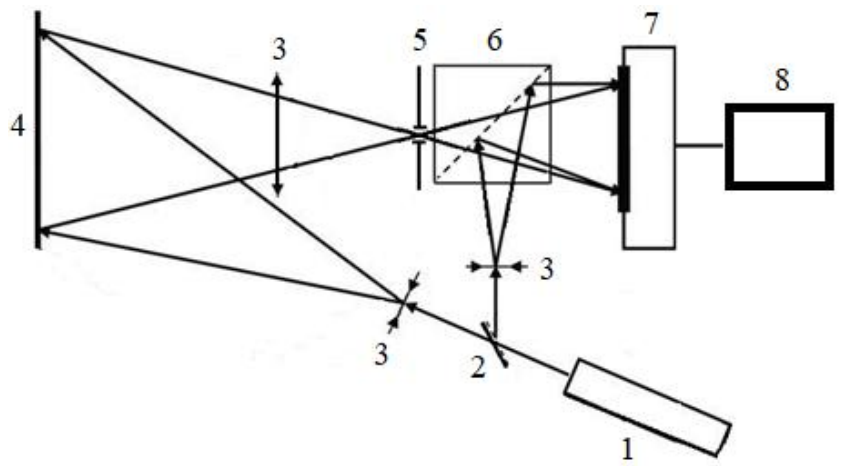

1 - лазер; 2 - делитель светового пучка; 3 - линза; 4 - наблюдаемая поверхность; 5 - диафрагма; 6 - делительный кубик; 7 - CCD-камера; 8 - компьютер

Рис. 3 - Принципиальная схема регистрации цифровых голограмм 
Последующая цифровая обработка таких голограмм с использованием, в частности, двойного Фурье-преобразования позволяет раздельно восстановить зарегистрированные на них распределения амплитуд и фаз в объектном (предметном) пучке. При этом фаза в каждой точке вычисляется как аргумент комплексного числа

$$
\varphi\left(x, y, z_{i}\right)=\operatorname{arctg} \frac{\operatorname{Im}\left[u_{0}\left(x, y, z_{i}\right)\right]}{\operatorname{Re}\left[u_{0}\left(x, y, z_{i}\right)\right]},
$$

где $u_{0}\left(x, y, z_{i}\right)$ - массив после второго Фурье-преобразования; $z_{i}$ - расстояние от приемного сенсора (голограммы) до рассматриваемой точки.

Результат вычитания фразовых распределений до и после нагружения образует интерферограмму, которая показывает распределение разности фраз по поверхности объекта, несложно пересчитываемое в распределение перемещений.

Для построения картины разности фраз, кроме двойного Фурьепреобразования, применяются и другие цифровые методы: трехпиксельный метод, прямое вычисление разности фраз (без построения интерферограммы) и интерференционные фрильмы. При этом распределение яркости интерференционных полос имеет разрывы в фразах $\pi / 2+\pi n$, которые затем устраняются с использованием алгоритма дециклизации. Полученное распределение чувствительно к направлению перемещения, чего не наблюдается в аналоговой двухэкспозиционной ГИ. Это сразу решает проблему определения знака перемещений и существенно облегчает расчет полной картины разности фраз в плоскости объекта.

Методы цифровой голографии основаны на использовании специализированных цифровых видеокамер в качестве устройства для регистрации голограмм. В настоящее время производители камер технического зрения предлагают широкий выбор изделий различного функционального назначения. Цифровые ПЗС-камеры с 10-40 миллионами пикселей и более являются стандартными. Их применение обеспечивает построение последовательности голограмм нестационарных перемещений практически в режиме реального времени. Попарным сравнением смежных голограмм из этой последовательности можно получать картину (интерференционный фильм) изменения перемещений во времени [6].

Использование камеры как устройства регистрации цифровых голограмм зависит от способа передачи данных от камеры к компьютеру, а также наличия возможности внешнего управления параметрами камеры, такими как внешний запуск, установка экспозиции, усиление, цветопередача и т. д. В частности, применение CCD-камеры Pulnix TM-1320$15 \mathrm{CL}$, современного ПК, программно-аппаратной среды Labview и программно-вычислительной среды Matlab дает возможность полностью совместить процессы регистрации с процессами обработки и представления результатов. 
Цифровые методы позволяют существенно расширить возможности голографических измерений, а именно - регистрировать существенно большие (до 100 мкм) перемещения, которые нельзя получить прямым методом двух экспозиций, и определять перемещения нанометрового диапазона. Первое достоинство является следствием того, что для вычисления интерферограммы большого перемещения, выходящего за пределы корреляционных свойств регистрирующей системы, можно использовать сумму его пошаговых голограмм, не выходящих за эти пределы - метод фазового сложения. Второе преимущество следует из того, что в цифровой голографии вычисляется, например, методом прямого расчета, распределение разности фаз между двумя состояниями объекта даже в том случае, когда на интерферограмме нет полос, и данную информацию можно использовать для определения поля перемещений.

Следует отметить, что регистрация голограмм методами цифровой голографии не только не дает какого-либо преимущества перед методами регистрации на фоточувствительные материалы (фотоэмульсии, фоототермопласт и т. п.), но и приводит к существенному снижению качества восстановленного изображения. Причина этого сильный спекл-шум, связанный с существенно меньшей разрешающей способностью цифровых видеокамер (до 400 лин/мм) по сравнению с классическими фоточувствительными средами для голографии (от 1000 до 5000 лин/мм, хотя в частных случаях достаточно 300 лин/мм).

С другой стороны, современные методы цифровой голографии значительно превосходят классические методы ГИ по таким параметрам, как чувствительность, помехозащищенность, быстрота получения конечного результата и наглядность его представления. Они легко встраиваются в автоматизированные измерительные комплексы и могут быть эффективно использованы как в лабораторных, так и в производственных условиях. Цифровая голография непрерывно развивается, расширяя диапазон измерений, возможности автоматизации, улучшая наглядность представления результатов.

Технологии на основе спекл-интерферометрии относятся к группе наиболее динамично развивающихся оптических высококогерентних методов анализа перемещений и деформаций поверхности конструкций, их элементов и материалов. В основе этих технологий лежит регистрация и последующий анализ хаотических пространственных интерференционных структур (спеклов), которые фрормируются диффузно отражающим объектом при его освещении монохроматическим когерентным светом $[17,18]$.

Спеклы - это результат интерференции света, отраженного от микрообластей рассеивающей поверхности, каждая из которых вносит свой вклад в фразу падающего на нее светового потока. В пространстве спекл имеет сигарообразную форму и ориентирован в сторону распространения; его размеры увеличиваются по мере удаления от поверхности. 


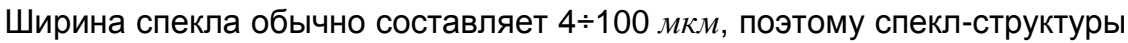
можно наблюдать и фиксировать фото- или видеокамерой.

Картина мгновенного распределения спеклов связана с микроструктурой (шероховатостью) и пространственным положением (полем перемещений) поверхности, а также разрешающей способностью системы, используемой для ее наблюдения. Она сдвигается с изменением точки освещения и точки наблюдения, зависит от расстояния до точки наблюдения и от скорости движения самой поверхности. Таким образом, наблюдаемая картина спеклов содержит информацию обо всех указанных фракторах. Способы регистрации и дешифровки таких картин составляют совокупность методов спекл-метрологии, из которых в исследованиях и диагностировании прочности конструкций и их элементов наибольшее применение находят методы СИ.

Методы СИ основаны на сложении (интерференции) предметного поля, имеющего спекл-структуру, с опорной волной в виде плоской либо сфрерической волны или другого поля со спекловой структурой. Практический интерес представляет СИ с использованием опорного поля со спекловой структурой, поскольку позволяет эффрективно исследовать трехмерные поля статических и динамических микроперемещений поверхности $[2,7,8,11,13,22]$. Ее разновидность - электронная спекл-интерферометрия (ЭСИ), базирующаяся на современных цифровых средствах регистрации суммарных спекл-картин и развитом программно-алгоритмическом обеспечении их обработки, называется также цифровой ЭСИ. К основным методам ЭСИ относятся корреляционная ЭСИ, временная фразосдвиговая ЭСИ, пространственная фазосдвиговая ЭСИ, ширография, ЭСИ с использованием Фурье-преобразования спекл-интерферограммы и гетеродинная ЭСИ.

Наиболее простым и распространенным методом ЭСИ является корреляционная ЭСИ, которая основана на определении взаимной корреляции двух спекл-интерферограмм, полученных при разных состояниях объекта, например, до и после его нагружения. Очевидно, что в этих состояниях распределения фазы предметного (объектного) поля и, следовательно, освещенности в результирующей спеклструктуре будут тоже разными.

Корреляционное сравнение спекл-интерферограмм обычно осуществляется путем их регистрации цифровым фотоприемником при фокусировке изображения на плоскость ПЗС-матрицы, последующего компьютерного попиксельного вычитания и отфильтровывания шумов на результирующей картине. В итоге участки изображения поверхности, где перемещения отсутствуют или отличаются на целое число полуволн используемого излучения, становятся темными, поскольку случайные функции их освещенности на сравниваемых спеклкартинах являются скоррелированными, то есть их разность минимальна. Соответственно участки, где эти функции максимально декоррелированы, становятся наиболее яркими. Таким образом, на экране дисплея формируется достаточно контрастная картина темных и светлых корреляционных полос (до 10 порядков), которая описывает 
изменение поля перемещений поверхности, обусловленное нагрузкой. Количественный анализ картины полос спекл-корреляции не содержит принципиальных затруднений, однако для упрощения этой процедуры регистрацию нормальной и тангенциальных компонент вектора перемещений осуществляют раздельно с использованием соответствующих по чувствительности интерферометров (рис. 4).

Корреляционная ЭСИ успешно использовалась для обнаружения расслоений в защитных покрытиях, исследования вибраций и статических деформаций деталей энергетических установок и определения перемещений у вершины трещины, при контроле микроперемещений поверхности, остаточных напряжений у круговых отверстий и в зонах сварных швов в натурных условиях, при оценке скорости деформирования растягиваемых образцов с отверстием и др.

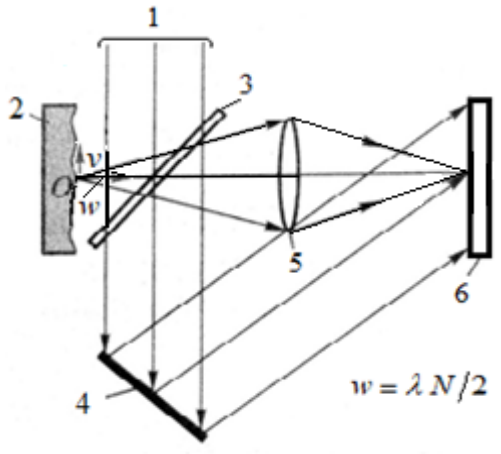

a

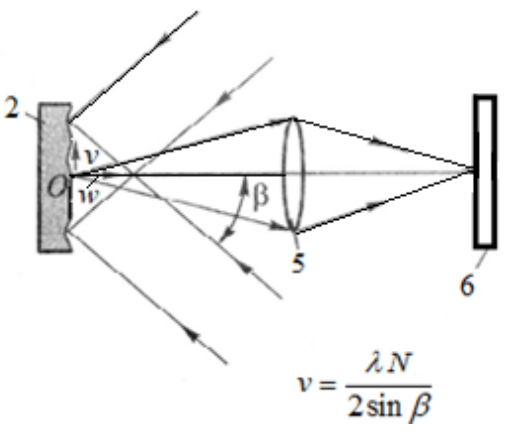

б

1 - лазер; 2 - исследуемая поверхность; 3 - делитель; 4 - диффрузное зеркало; 5 - объектив; 6 - регистратор; $N$ - порядок светлой полосы корреляции

Рис. 4 - Схемы определения нормальной (а) и тангенциальной (б) компонент вектора перемещения методом корреляционной спекл-интерферометрии

Корреляционная ЭСИ успешно использовалась для обнаружения расслоений в защитных покрытиях, исследования вибраций и статических деформаций деталей энергетических установок и определения перемещений у вершины трещины, при контроле микроперемещений поверхности, остаточных напряжений у круговых отверстий и в зонах сварных швов в натурных условиях, при оценке скорости деформирования растягиваемых образцов с отверстием и др.

Метод позволяет измерять перемещения поверхности величиной до $5 \lambda$ с погрешностью не менее $\lambda / 2$. Его существенным преимуществом является простота реализации реально-временного варианта, т. е. возможности изучения динамического деформирования поверхности на экране монитора.

Методы временной фазосдвиговой ЭСИ по сравнению с корреляционной ЭСИ являются более сложными, однако дают возможность 
существенно повысить точность измерения микро- и наноперемещений поверхности. Во временной фазосдвиговой ЭСИ для определения величин и разницы фаз интерференционных полос до и после нагружения используются различные фазосдвиговые устройства и алгоритмы. Но для каждого состояния поверхности пошагово формируют несколько (до пяти) спекл-интерферограмм, при этом поверхность должна оставаться неподвижной.

Корректное использование временной фразосдвиговой ЭСИ возможно лишь при определении статических перемещений поверхности. Теоретически ее чувствительность к перемещениям может быть очень высокой, на практике же достигает значений $\lambda / 300$.

В пространственной фазосдвиговой ЭСИ для определения разницы фаз интерференционных полос до и после нагружения все сдвинутые по фразе с малым шагом спекл-интерферограммы регистрируются практически одновременно, что нейтрализует влияние вибрации и большинства других движений поверхности как жесткого целого на результаты измерений. Эту интерферометрию можно применять в производственных условиях.

Методы временной и пространственной фазосдвиговой ЭСИ широко используется для решения задач экспериментальной механики. Известны их применения в измерении параметров разрушения изгибаемого балочного образца бетона, армированного стальными волокнами, раскрытия трещин в бетонных образцах с центральным надрезом при определении коэфрфициента интенсивности напряжений $K_{\mathrm{I}}$ в образцах из различных конструкционных материалов с краевой поперечной трещиной при изгибе, для оценки деформаций на краю пластины из углепластика при изгибе и полей трехмерных деформаций поверхности коробки передач автомобиля. Методы показали также хорошую эффективность в технической диагностике элементов и материалов конструкций, в частности при контроле сотовых структур, сварных швов, внутренние трещины в трубопроводах и др.

Ширография (от англ. shear - сдвиг) является наиболее интенсивно развивающимся когерентно-оптическим методом исследования и контроля конструкций на основе алгоритмов фазосдвиговой ЭСИ [24]. Его суть состоит в следующем. Тестируемый шероховатый объект частично или полностью освещается когерентным светом, который при отражении попадает на сдвиговый элемент (например, бипризма Френеля), размещенный перед объективом ССД-камеры. После сдвигового элемента фрормируются два поперечно сдвинутые изображения (спекл-поля), которые интерферируют друг с другом, образуя пространственную хаотическую микроинтерференционную картину. Слой этой картины, оказавшийся в плоскости матрицы на основе приборов зарядовой связи (ПЗС-матрицы) ССД-камеры, регистрируется и вводится в компьютер.

Полученные микроинтерференционные спекл-картины, записанные для двух состояний (до и после изменения параметров нагружения), 
сравниваются и обрабатываются по специальной программе. В результате на экране монитора появляется изображение наблюдаемой поверхности с четкими макроинтерференционными темными и светлыми полосами или широграмма. Широграмма несет информацию о деформационных смещениях поверхности от нагрузки в виде производных от компонент вектора перемещений ее точек в направлении сдвига. Поэтому зоны концентрации деформаций и напряжений разделяются на меньшие смежные области, соответственно с положительными и отрицательными углами наклона местного рельефа поля перемещений; формы колебаний поверхности также необычны - в виде уровней величин указанных производных.

Ширография позволяет оперативно выявлять перемещения поверхности с хорошим разрешением (несколько микрометров), не требует использования специальных устройств или сложных сканирующих механизмов. Согласно ширографической технологии интерферирующие пучки света проходят до сдвигового элемента по одному и тому же каналу. Это обеспечивает методу нечувствительность к вибрациям объекта как жесткого целого и позволяет использовать его в промышленных и полевых условиях. Метод применялся в измерении деформаций конструкций и их элементов при колебаниях и статическом нагружении, может использоваться для отображения действующих напряжений.

В последние годы ширография находит эффективное и возрастающее применение в диагностике механических свойств и других показателей качества оболочечно-пластинчатых конструкций, изготовленных из металлических и композиционных материалов. Это - элементы и узлы авиационных и космических конструкций (сотовые панели, тонколистовые металлические панели, полученные с использованием точечной сварки, тонкостенные трубы из стеклопластика, оболочки из углеродных композиционных материалов), автомобильные шины, печатные платы и др. В диагностических обследованиях используются, как правило, компактные ширографические установки.

К недостаткам метода следует отнести высокую стоимость выпускаемых ширографических систем (до \$100 000 при площади охватываемой видеокамерой сцены до $1 \mathrm{~s}^{2}$ ), а также их ограничения по кривизне контролируемой поверхности (квазиплоские участки).

Методы ЭСИ с использованием Фурье-преобразования спеклинтерферограммы рассматриваются как одно из направлений пространственной фазосдвиговой ЭСИ и используют при исследовании динамических деформаций, однако их чувствительность на порядок меньше чувствительности временной фразосдвиговой ЭСИ.

В гетеродинной ЭсИ перемещения объекта определяются путем модуляции длины волны и интенсивности используемого лазерного излучения различными способами. В частности, исследуемая поверхность освещается двумя пучками с определенной разницей хода с двух противоположных сторон под одинаковым углом (рис. 4); перемещения определяются путем анализа интерференции этих пучков после отражения. 
Метод дает возможность измерять перемещения величиной до 200 мкм с погрешностью $\sim 1$ ни и поэтому используется в прецизионных измерениях и мониторинге статических деформаций и вибраций элементов микроконструкций.

Методы СИ не предъявляют жестких условий к разрешающей способности регистрирующей среды, имеют хорошие показатели чувствительности, точности и оперативности измерений при пониженной чувствительности к внешним помехам. Возможности практического приложения методов возрастают в связи с внедрением в исследования новых более совершенных средств и технологий цифровой регистрации и обработки интерферограмм.

Современные варианты методов классической интерферометрии. Методы оптической интерферометрии применяются при решении задач технической диагностики микро- и нанорельефа поверхностей конструкций, их элементов и материалов. Как известно, надежное и точное определение начала и развития локальных изменений рельефа поверхности контролируемого образца на этих уровнях имеет большое значение в прогнозировании его прочности и ресурса, особенно при циклических нагрузках.

Современные методы оптической интерферометрии и соответствующие измерительные системы, сохраняя принципы и схемы классической интерферометрии, базируются на высококогерентных (лазерных) и низкокогерентных (ламповых и др.) устройствах освещения, скоростных компьютерах и цифровых видеокамерах с матричными мегапиксельными сенсорами для регистрации изображений, высокоэффрективном программно-алгоритмическом обеспечении для обработки интерференционных картин $[5,12,23]$. Качественный скачок в развитии методологии и технического оснащения оптической интерферометрии позволил значительно повысить скорость и точность обработки наблюдаемых картин по сравнению с ранее применявшимися технологиями на основе визуального анализа.

В настоящее время наиболее распространенными являются методы лазерной одноволновой, двухволновой и многоволновой интерферометрии, сканирующей интерферометрии в белом свете и интерферометрии с непрерывным изменением длины волны.

В методах лазерной одноволновой интерферометрии (временная и пространственная фразосдвиговая интерферометрия) используются различные фазосдвиговые алгоритмы, которые обеспечивают высокую точность измерения фазы интерференционных полос и, следовательно, нанорельефа наблюдаемой поверхности. Временную фазосдвиговую интерферометрию применяют в диагностировании поверхности в ее статическом состоянии. Разрешающая способность метода может достигать 0,3 нм при максимальных величинах перепада высот рельефа $\leq \lambda / 4$. Пространственная фазосдвиговая интерферометрия не предъявляет строгих требований к стабильности картин полос в процессе измерений и поэтому применяется в неразрушающем контроле и технической 
диагностике элементов конструкций при их неустановившемся деформировании и вибрации. Однако при таких же максимальных перепадах высот рельефа разрешающая способность метода значительно ниже ( 30 нм).

Возможность значительно расширить диапазон контролируемых высот рельефа дают методы лазерной двух- и многоволновой интерферометрии, но они сложнее в реализации.

Методы сканирующей интерферометрии в белом свете основаны на сравнении длин оптических путей предметного (отраженного от поверхности) и опорного низкокогерентных пучков в двулучевых интерферометрах. В данном случае интерференционные полосы формируются только в очень узкой $(1 \div 2$ мкм) зоне пространства у исследуемой поверхности в виде синусоидального цуга (карелограммы). Полоса 0-го порядка карелограммы (плоскость, в которой оптическая разность хода сравниваемых лучей равна нулю), пересекая рельеф поверхности, образует на ней наиболее контрастную интерференционную полосу. Путем механического сканирования поверхности по нормали к ней, регистрации на каждом шаге этой наиболее контрастной полосы и обработки серии полученных интерферограмм формируется топограмма поверхности. Сканирующая интерферометрия в белом свете применяется в измерениях нанорельефа поверхности с достаточно высокой разрешающей способностью в направлении сканирования ( 0,3 нм), при этом ширина диапазона высот микронеровностей достигает 460 нм.

В интерферометрии с непрерывным изменением длины волны используются источники излучения с перестраиваемой частотой (лазеры на красителях, твердотельные и диодные лазеры с перестраиваемой частотой). Как и в сканирующей интерферометрии в белом свете, здесь тоже регистрируется ряд интерферограмм контролируемой поверхности, но сканирование рельефа по высоте происходит автоматически, поскольку пространственная частота интерференционных полос изменяется синхронно с изменением длины волны. Разрешающая способность метода относительно низкая ( 0,5-1,0 мкм).

Практически все методы современной оптической интерферометрии, выигрывая в скорости и точности измерения микро- и нанорельефа, сохраняют свою высокую эффективность лишь применительно к малогабаритным объектам с плоской оптически гладкой или наношероховатой поверхностью контроля (элементы микро- и оптоэлектроники, микроэлектромеханических систем, кристаллы и т.п.).

Выводы. Представленный обзор может быть полезным при планировании исследований и неразрушающего контроля неоднородных конструкций, их элементов и материалов. От совместного применения рассмотренных интерференционно-оптических методов, а также их комплексного использования с методом конечных элементов для расчета напряжений следует ожидать повышения эффективности исследований и контроля. 


\section{БИБЛИОГРАФИЧЕСКИЕ ССЫЛКИ}

1. Вест Ч. Голографическая интерферометрия; пер. с англ. М.: Мир, 1982. 504 с.

2. Горбатенко Б. Б., Рябухо В. П., Гребенюк А. А. и др. Контроль микроперемещений методами цифровой голографической и спекл-интерферометрии // Вестн. Саратовского гос. ун-та. 2010. Т. 4. № 1. С. 14-21.

3. Гудрамович В. С., Дзюба А. П., Селиванов Ю. М. Методы голографической интерферометрии в механике неоднородных тонкостенных конструкций: монография. Д.: Лира, 2017. 288 с.

4. Гудрамович В. С., Скальський В. Р., Селіванов Ю. М. Голографрічне і акустико-емісійне діагностування неоднорідних конструкцій і матеріалів:монографія /. За заг. ред. 3. Т. Назарчука. Львів: Простір-М, 2017. 492 с.

5. Гужов В. И., Ильиных С. П. Компьютерная интерферометрия. Новосибирск: Изд. НГТУ, 2004. 252 с.

6. Гуревич В. С., Гусев М. Е., Гапонов В. Е., Редкоречев В. И. Цифровая голографическая интерферометрия как метод анализа деформаций. Современное состояние и перспективы развития // Сб. трудов 7-й Междунар. научно-практич. конф. «Голография - наука и практика» 28-30 сентября 2010, Москва. С. 350-357.

7. Каленко Г., Штанько А. Цифровая корреляционная спекл-интерфрерометрия // Фотоника. 2010. № 4. С. 58-60.

8. Крюков Д. Б., Розен А. Е., Соловьев В. А., Лось И. С. Метод корреляционной спекл-интерферометрии для контроля биметаллов, полученных сваркой, взрывом // Изв. Волгоградского гос. техн. ун-та. 2006. № 9. С. 76-79.

9. Ларіонова О. О., Рожковський В. Ф., Сохач Ю. В. Голографічні технології в авіаційно-космічній техніці: навч. посіб./ Під ред. В. П. Малайчука. Д.: ДНУ, 2003. 272 с.

10. Лобанов Л. М., Знова В. А., Пивторак В. А., Киянец И. В. Технология неразрушающего контроля качества элементов и узлов авиационных конструкций методом электронной ширографии // Техн. диагностика и неразрушающий контроль. 2014. № 3. С. 15-19.

11. Минеев С. А., Угольников А. Ю., Лозовская Л. Б. Анализ спекл-изображений деформируемой поверхности на основе алгоритмов обработки оптического потока // Вестн. Нижегородского ун-та. Сер.: Радиофизика. 2014. № 2 (1). С. 81-86.

12. Муравський Л. І., Вороняк Т. І., Кметь А. Б. Лазерна інтерферометрія поверхні для потреб технічної діагностики. Львів: Сполом, 2014. 272 с.

13. Муравський Л. І. Методи спекл-кореляції для дослідження механічних властивостей конструкційних матеріалів. К.: Наукова думка, 2010. 208 с.

14. Островский Ю. И., Щепинов В. П., Яковлев В. В. Голографические интерференционные методы измерения деформаций. М.: Наука, 1988. 248 с.

15. Селиванов Ю. М. Применение комбинированных голографических интерферограмм к исследованию неустановившихся деформаций // Доп. НАН України. Сep.: A. 2007. № 6. C. 56-60.

16. Baltisky S., Gurov J., De Nivola S. et. al. Characterization of microelectromechanical systems by the digital holography method // Imaging Sci. J. 2006. Vol. 54. No. 2. P. 103-110.

17. Goodman J. W. Speckle Phenomena in Optics: Theory and Applications. Greenwood Village, CO: Roberts and Company, 2007. 388 p.

18. Interferometry in Speckle Light: Theory and Applications. Eds. P. Jacquot, J.-M. Fournier. Berlin: Springer, 2000. 660 p.

19. Kreis $\boldsymbol{T}$. Handbook of Holographic Interferometry: Optical and Digital Methods. WILEYVCH Verlag Gmbh \& Co., 2005. 542 p.

20. Pedrini G., Osten W., Gusev M. E. High-speed digital holographic interferometry for vibration measurement // Appl. Opt. 2015. Vol. 45. No 15. P. 3456-3462. 
21. Schnars U., Jueptner W. Digital Holography: Digital Hologram Recording, Numerical Reconstruction, and Related Techniques. Dordrecht: Springer, 2005. 172 p.

22. Shchepinov V. P., Pisarev V. S. et al. Strain and Stress Analysis by Holographic and Speckle Interferometry. Chichester: John Wiley \& Sons, 1996. 496 p.

23. Sirohi S. R. Optical Methods of Measurement: Wholefield Techniques, 2-nd ed. Boca Raton, FL: Taylor \& Francis, 2009. 290 p.

24. Steinchen W., Yang L. Digital Shearography: Theory and Application of Digital Speckle Pattern Shearing Interferometry // SPIE Press. PM 100. Bellingham, WA: SPIE, 2003. 230 p.

УДК 535.31, 621.658.011

Ю. М. Селіванов, д-р техн. наук, С. С. Кузьонний

\section{СУЧАСНІ ІНТЕРФЕРЕНЦІЙНО-ОПТИЧНІ ТЕХНОЛОГІЇ ДОСЛІДЖЕННЯ ТА НЕРУЙНІВНОГО КОНТРОЛЮ НЕОДНОРІДНИХ КОНСТРУКЦІЙ (ОГЛЯД)}

Розглянуто стан технологій дослідження й неруйнівного контролю міцності та динамічних властивостей неоднорідних конструкцій на основі голографічної інтерферометрії, спекл-інтерферометрії, висококогерентної і низкокогерентної класичної інтерферометрії. Наведено їх особливості, області ефективного застосування.

Ключові слова: голографрічна інтерферометрія, спекл-інтерферометрія, класична інтерферометрія, нові технології, області застосування.

UDC 535.31, 621.658.011

Yu. M. Selivanov, Dr. Sci. (Tech.), S. S. Kuzenniy

\section{MODERN INTERFERENCE-OPTICAL RESEARCH TECHNOLOGY AND NON-DESTRUCTIVE TESTING OF INHOMOGENEOUS STRUCTURES (REVIEW)}

The state of research technologies and non-destructive testing of strength and dynamic properties of inhomogeneous structures on the basis of holographic interferometry, speckle interferometry, high-coherence and low-coherence classical interferometry is considered. Their features, areas of effective application are given.

Keywords: holographic interferometry, speckle interferometry, classical interferometry, new technologies, areas of use.

In recent decades, in research and non-destructive control of inhomogeneous structures, predominantly digital technologies based on interference-optical methods are increasingly used - holographic interferometry $(\mathrm{HI})$, speckle interferometry (SI), high-coherence and low-coherence classical interferometry [1 - 24]. The review contains the current state of these technologies.

Methods of holographic interferometry are considered the most developed and widely used in research and non-destructive testing of heterogeneous thin-walled structures $[1,3,4,9,14,19,22]$. Double-exposed $\mathrm{HI}$ and time-averaged $\mathrm{HI}$ are most often used, less often - stroboscopic $\mathrm{HI}$ and $\mathrm{HI}$ in 
real time. The method of combined holographic interferograms [3, 15] and digital $\mathrm{HI}[2,6,16,19-21]$ are increasingly used.

Attractive general advantages of $\mathrm{HI}$ methods are the possibility of simultaneous observation of the surface area of $10 \mathrm{sq} . \mathrm{m}$. and more, as well as the absence of fundamental restrictions on the shape, condition of the surface and material of the tested object. Their use allows to receive without contact qualitative and accurately enough quantitative information about static and nonstationary fields of micro- and nanomovement of the surface of the controlled structure under the influence of various factors; the range of recorded displacements - from units of nanometers to 100 micrometers. By these movements it is possible to evaluate the stress-strain state of the structure, to identify its structural imperfections, technological defects, operational damage, to control the process of crack development, etc. However, in order to implement $\mathrm{HI}$ methods, a high-resolution registering environment (when using classical or analogue $\mathrm{HI}$ ) or a matrix light-sensitive sensor with 10-40 million pixels and the corresponding hardware and software environment (in case of using digital $\mathrm{HI}$ ) is required.

$\mathrm{HI}$ methods continue to be developed and improved, and new practical applications are found. This section presents some new elaborations in the field of holographic technologies, in particular, the method of analysis of interferograms by the indicated bands density, the method of combined holographic interferograms used to research and control structures in their nonstationary states, etc.

Speckle interferometry provides for the registration and subsequent analysis of chaotic spatial interference structures (speckles), which are formed by a diffusely reflecting object under its illumination by monochromatic coherent light $[11,17,18]$. The picture of instantaneous distribution of speckles depends on the microstructure (roughness) and spatial position (field of movement) of the surface, its velocity, etc. One of the leading directions of measuring instruments is electronic speckle interferometry (ESI), which is based on modern digital means of speckle-picture registration and developed software and algorithmic software for their processing, also called digital ESI.

SI methods are based on the interference (addition) of the subject field and reference wave, which, as a rule, have different speckle-structures. Modern SI methods include correlation ESI, temporary phase-shifting ESI, spatial phase-shifting ESI, shearography, ESI with using Fouriertransformations of interferograms and heterodyne ESI. Their application makes it possible to effectively research three-dimensional fields of static and dynamic surface micro-movements [2, 7, 8, 13, 22].

Correlation ESI allows to measure surface movements of up to $5 \lambda$ with an error of at least $\lambda / 2$. Its essential advantage is the simplicity of implementation of the real-time variant, i.e. the possibility of studying the dynamic surface deformation on the monitor screen. The method was successfully used for investigation of vibrations and static deformations of parts, detection of stratification in the protective coatings, determination of movements at the top of the crack, control of residual stress at circular holes and in the zones 
of welds in field conditions, in the evaluation of the rate of deformation of stretched samples with a hole, etc.

Methods of temporal phase-shifting ESI are more complex, but they allow to significantly increase the accuracy of measurements of static microand nano-surface displacements. In the time phase shift ESI, different phase-shifting devices and algorithms are used to determine the values and phase differences of the interference bands before and after loading. But for each state of the surface, several (up to five) speckle interferograms are formed step by step, and the surface must remain stationary. Sensitivity of the temporary phase-shifting ESI to movements reaches $\sim 2 \mathrm{~nm}$.

In the spatial phase-shifting ESI to determine the phase difference of interference bands before and after loading, all the phase shifted speckleinterferograms with a small step are registered almost simultaneously, which neutralizes the effect of vibration and most other surface motions as a rigid whole on the measurement results.

Methods of temporary and spatial phase-shifting ESI are widely used to solve the problems of experimental mechanics, have also shown good efficiency in technical diagnostics of elements and materials of structures, in particular in the control of cellular structures, welds, internal cracks in pipelines, etc.

Shearography is the most intensively developing coherent-optical method of investigation and control of structures on the basis of phaseshifting ESI algorithms [10, 24]. The method allows to detect surface movements with good resolution (several micrometers). Shearography was used in measuring deformations of structures and their elements under vibration and static loading. It finds effective and increasing application in diagnostics of mechanical properties and other indicators of quality of shell-and-plate constructions made of metal and compositional materials. The disadvantages of the method include the high cost of manufactured shearographical systems, as well as their limitations on the curvature of the controlled surface (quasiplane areas).

In the spatial phase-shifting ESI to determine the phase difference of interference bands before and after loading, all the phase shifted speckleinterferograms with a small step are registered almost simultaneously, which neutralizes the effect of vibration and most other surface motions as a rigid whole on the measurement results.

Methods of temporary and spatial phase-shifting ESI are widely used to solve the problems of experimental mechanics, have also shown good efficiency in technical diagnostics of elements and materials of structures, in particular in the control of cellular structures, welds, internal cracks in pipelines, etc.

Shearography is the most intensively developing coherent-optical method of investigation and control of structures on the basis of phaseshifting ESI algorithms [10, 24]. The method allows to detect surface movements with good resolution (several micrometers). Shearography was used in measuring deformations of structures and their elements under vibration and static loading. It finds effective and increasing application in diagnostics 
of mechanical properties and other indicators of quality of shell-and-plate constructions made of metal and compositional materials. The disadvantages of the method include the high cost of manufactured shearographical systems, as well as their limitations on the curvature of the controlled surface (quasiplane areas).

Laser single-wave interferometry methods provide high accuracy of nano-relief measurements of the observed surface. In the static state of the surface the resolution $\Delta h$ can reach $0,3 \mathrm{~nm}$ at the maximum values of the difference in elevation $\Delta H \leq \lambda / 4$ ( $\lambda$ - wavelength), in the non-stationary state at the same resolution is much lower $(\approx 30 \mathrm{~nm})$.

Scanning white light interferometry methods are used for surface nanorelief measurements with high resolution in the direction of normal to the surface $(\Delta h \approx 0,3 \mu м)$, where $\Delta H \leq 460 \mu м$.

Almost all modern varieties of classical interferometry methods, winning in the speed and accuracy of micro- and nano-relief measurements, retain their high efficiency only with respect to small objects with a plane optically smooth or nano-coated control surface (elements of micro- and optoelectronics, microelectromechanical systems, crystals, etc.).

Relatively wide range of displacements measured by interferenceoptical methods in general (from fractions of $\mathrm{nm}$ to 100 microns) at their high sensitivity and increasing level of computerization of interferograms obtaining and processing increase interest of specialists in the field of nondestructive control and technical diagnostics to them, open up prospects of effective application in researches.

\section{REFERENCES}

1. West Ch. Holographic Interferometry. Moscow: Mir, 1982. 504 p. (in Russian).

2. Gorbatenko B. B., Ryabuho V. P., Grebenyuk A. A. et al. Control of micromovements by the methods of the digital holographic and speckle-interferometry // Vestnik of Saratov states un-t. 2010. V. 4. No. 1. P. 14-21. (in Russian).

3. Gudramovych V. S., Dzyuba A. P., Selivanov Yu. M. Methods of holographic interferometry in the mechanics of heterogeneous thin-walled structures: monograph, Dnepr: Lyra, 2017. 288 p. (in Russian).

4. Gudramovych V. S., Skalskyi V. R., Selivanov Yu. M. Holographic and acoustic emission diagnostics of inhomogeneous structures and materials: monograph / ed. by acad. of NAS Ukraine Z. T. Nazarchuk. Lviv: "Prostir-M", 2017. 492 p. (in Ukrainian).

5. Guzhov V. I., llyinykh S. P. Computer interferometry. Novosibirsk: Publishing house NGTU, 2004. 252 p. (in Russian).

6. Gurevich V. S., Gusev M. E., Gaponov V. E., Redkorechev V. I. Digital holographic interferometry as a method of deformation analysis. Current state and prospects of development // Proc. of the 7th Int. Scien.-Pract, Conf. "Holography - Science and Practice". September 28-30 2010, Moscow. P. 350-357. (in Russian).

7. Kalenko G., Shtanko A. Digital correlation speckle-interferometry // Photonics. 2010. No. 4. P. 58-60. (in Russian).

8. Kryukov D. B., Rosen A. E., Soloviev V. A., Moose I. S. Correlation speckleinterferometry method for control of bimetals obtained by explosion welding // Izvestia of Volgograd states u-t. 2006. No. 9. P. 76-79. (in Russian). 
9. Larionova O. O., Rozhkovsky V. F., Sohach Yu. V. Holographic technologies in aerospace engineering: textbook. Tool / Ed. V. P. Malaychuk. Dnepropetrovsk: RVV DNU, 2003. 272 p. (in Ukrainian).

10. Lobanov L. M., Znova V. A., Pivtorak V. A., Kiyanets I. V. Technology of nondestructive quality control of elements and units of aviation structures by the method of electronic shearography // Technological diagnostics and nondestructive testing. 2014. No. 3. P. 15-19. (in Russian).

11. Mineyev S. A., Ugolnikov A. Y., Lozovskaya L. B. Analysis of the speckle-images of the deformed surface on the basis of the optical flow processing algorithms // Vestnik Nizhegorod un-t. Ser.: Radiophysics. 2014. No. 2 (1). P. 81-86. (in Russian).

12. Muravskiy L. I., Voroniak T. I., Kmeti A. B. Laser interferometry of the surface for the need of technical diagnostics. Lviv: Spolom, 2014. 272 p. (in Ukrainian).

13. Muravsky L. I. Speck-correlation methods for the study of mechanical properties of structural materials. Kyiv.: Naukova dumka, 2010. 208 p. (in Ukrainian).

14. Ostrovsky Yu. I., Shchepinov V. P., Yakovlev V. V. Holographic interference methods of deformation measurement. Moscow: Nauka. 248. p. (in Russian).

15. Selivanov Yu. M. Application of the combined holographic interferograms to the study of the nonstationary deformations // Reports of the NAS of Ukraine. Seri A. 2007. No.6. P. 56 - 60. (in Russian).

16. Baltisky S., Gurov J., De Nivola S. et. al. Characterization of microelectromechanical systems by the digital holography method // Imaging Sci. J. 2006. Vol. 54. No. 2. P. $103-110$.

17. Goodman J. W. Speckle Phenomena in Optics: Theory and Applications. Greenwood Village, CO: Roberts and Company, 2007. 388 p.

18. Interferometry in Speckle Light: Theory and Applications / Eds. P. Jacquot, J.-M. Fournier. Berlin: Springer, 2000. 660 p.

19. Kreis T. Handbook of Holographic Interferometry: Optical and Digital Methods. WILEY-VCH Verlag Gmbh \& Co., 2005. 542 p.

20. Pedrini G., Osten W., Gusev M. E. High-speed digital holographic interferometry for vibration measurement // Appl. Opt. 2015. Vol. 45. No. 15. P. 3456-3462.

21. Schnars U., Jueptner W. Digital Holography: Digital Hologram Recording, Numerical Reconstruction, and Related Techniques. Dordrecht: Springer, 2005. 172 p.

22. Shchepinov V. P., Pisarev V. S. et al. Strain and Stress Analysis by Holographic and Speckle Interferometry. Chichester: John Wiley \& Sons, 1996. 496 p.

23. Sirohi S. R. Optical Methods of Measurement: Wholefield Techniques, 2-nd ed. Boca Raton, FL: Taylor \& Francis, 2009. 290 p.

24. Steinchen W., Yang L. Digital Shearography: Theory and Application of Digital Speckle Pattern Shearing Interferometry // SPIE Press. - PM 100. Bellingham, WA: SPIE, 2003. 230 p.

Дніпровський національний

університет імені Олеся Гончара,

Дніпро, Україна

Надійшла до редколегії 06.05.2019 